\title{
A CONSTRUCTION OF INFINITE SETS OF INTERTWINES FOR PAIRS OF MATROIDS
}

\author{
JOSEPH E. BONIN
}

\begin{abstract}
An intertwine of a pair of matroids is a matroid such that it, but none of its proper minors, has minors that are isomorphic to each matroid in the pair. For pairs for which neither matroid can be obtained, up to isomorphism, from the other by taking free extensions, free coextensions, and minors, we construct a family of rank- $k$ intertwines for each sufficiently large integer $k$. We also treat some properties of these intertwines.
\end{abstract}

\section{INTRODUCTION}

If the classes $\mathcal{C}_{1}$ and $\mathcal{C}_{2}$ of matroids are minor-closed, then so is $\mathcal{C}_{1} \cup \mathcal{C}_{2}$. If $M$ is an excluded minor for $\mathcal{C}_{1} \cup \mathcal{C}_{2}$, then some minor of $M$ is an excluded minor for $\mathcal{C}_{1}$ and another is an excluded minor for $\mathcal{C}_{2}$; furthermore, no proper minor of $M$ has this property. These remarks motivate the following definition. A matroid $M$ is an intertwine of matroids $M_{1}$ and $M_{2}$ if $M$ but none of its proper minors has both an $M_{1}$-minor (i.e., a minor isomorphic to $M_{1}$ ) and an $M_{2}$-minor. Thus, each excluded minor for $\mathcal{C}_{1} \cup \mathcal{C}_{2}$ is an intertwine of some excluded minor for $\mathcal{C}_{1}$ and some excluded minor for $\mathcal{C}_{2}$.

Many important results and problems in matroid theory involve the question of whether the set of excluded minors for a given minor-closed class of matroids is finite; this leads to the question of whether some pairs of matroids have infinitely many intertwines. This question was raised by Tom Brylawski [2]; see also [5, Problem 14.4.6], where it is also attributed to Neil Robertson and, in a different form, to Dominic Welsh. The question was settled affirmatively by Dirk Vertigan in the mid 1990's in unpublished work; we sketch his construction in Section 5 Jim Geelen gave another construction [3, Section 5]: for each pair of spikes, neither being a minor of the other and all elements of which are in dependent transversals, he constructed infinitely many intertwines that are also spikes. (That the class of spikes contains such infinite sets of intertwines follows from Vertigan's construction along with his embedding of the minor ordering on all matroids into that on the class of spikes (for this intriguing embedding, see [3, Section 3]); Geelen's construction is an attractive realization of this phenomenon.) In this paper, we take weaker hypotheses than the earlier constructions used; we assume only that neither $M_{1}$ nor $M_{2}$ can be obtained, up to isomorphism, from the other via free extensions, free coextensions, and minors; for such a pair $\left(M_{1}, M_{2}\right)$, we show that particular amalgams of certain free coextensions of $M_{1}$ and $M_{2}$ are intertwines. This yields many intertwines of each sufficiently large rank; indeed, for some pairs, a variation on our basic construction produces intertwines whose number grows at least exponentially as a function of the rank.

We assume readers know basic matroid theory, an excellent account of which is in [5]. Key background topics are collected in Section 2 and the construction and a variation are

Date: October 28, 2018.

1991 Mathematics Subject Classification. Primary: 05B35.

Key words and phrases. Matroid, intertwine, cyclic flat, free extension, free coextension. 
given in Section 3. In Section 4, we treat properties of these intertwines; for instance, we show that for large ranks, the intertwines we construct have large connectivity and uniform minors of large rank and corank; we show that if both matroids have no free elements, no cofree elements, no isthmuses, and no loops, then, for a fixed integer $k$, the intertwines we construct cover the full range of possible sizes for the ground sets of rank- $k$ intertwines of the pair; we also show that the construction preserves certain properties, such as being transversal and being a gammoid. In Section 5, we explain the relation between our construction and Dirk Vertigan's.

\section{BACKGROUND}

The intertwines we construct are defined via cyclic flats and their ranks. A cyclic set in a matroid $M$ is a (possibly empty) union of circuits. The cyclic flats of $M$, ordered by inclusion, form a lattice; indeed, $F \vee G=\operatorname{cl}_{M}(F \cup G)$ and $F \wedge G$ is the union of the circuits in $F \cap G$. We let $\mathcal{Z}(M)$ denote both the set and the lattice of cyclic flats of $M$. The following well-known results are easy to prove [5], Problem 2.1.13]:

(1) $\mathcal{Z}\left(M^{*}\right)=\{S-F: F \in \mathcal{Z}(M)\}$, where $S$ is the ground set of $M$,

(2) $\mathcal{Z}\left(M_{1} \oplus M_{2}\right)=\left\{F_{1} \cup F_{2}: F_{1} \in \mathcal{Z}\left(M_{1}\right)\right.$ and $\left.F_{2} \in \mathcal{Z}\left(M_{2}\right)\right\}$, and

(3) a matroid is determined by its cyclic flats and their ranks.

There are many ways to prove property (3); for instance, one can show how to get the circuits or the independent sets, or show that the rank of an arbitrary set $Y$ in $M$ is given by the formula

$$
r(Y)=\min \{r(F)+|Y-F|: F \in \mathcal{Z}(M)\} .
$$

The following result from [7, 1] carries property (3) further.

Proposition 2.1. Let $\mathcal{Z}$ be a collection of subsets of a set $S$ and let $r$ be an integer-valued function on $\mathcal{Z}$. There is a matroid for which $\mathcal{Z}$ is the collection of cyclic flats and $r$ is the rank function restricted to the sets in $\mathcal{Z}$ if and only if

(Z0) $\mathcal{Z}$ is a lattice under inclusion,

(Z1) $r\left(0_{\mathcal{Z}}\right)=0$, where $0_{\mathcal{Z}}$ is the least element of $\mathcal{Z}$,

(Z2) $0<r(Y)-r(X)<|Y-X|$ for all sets $X, Y$ in $\mathcal{Z}$ with $X \subset Y$, and

(Z3) for all pairs of incomparable sets $X, Y$ in $\mathcal{Z}$,

$$
r(X)+r(Y) \geq r(X \vee Y)+r(X \wedge Y)+|(X \cap Y)-(X \wedge Y)| .
$$

Recall that the free extension $M+x$ of the matroid $M$ on $S$ by the element $x \notin S$ is the matroid on $S \cup x$ whose circuits are those of $M$ along with the sets $B \cup x$ as $B$ runs over the bases of $M$. We extend this notation to sets: $M+X$ is the result of applying free extension iteratively to add all elements of $X$ to $M$. From the perspective of Proposition 2.1 $M+X$, for $X \neq \emptyset$, is the matroid on $S \cup X$ whose cyclic flats and ranks are (i) the proper cyclic flats $F$ of $M$, with rank $r_{M}(F)$, and (ii) $S \cup X$, of rank $r(M)$. Dually, the cyclic flats and ranks of the free coextension $M \times X=\left(M^{*}+X\right)^{*}$ are (i) the sets $F \cup X$, of rank $r_{M}(F)+|X|$, for $F \in \mathcal{Z}(M)$ with $F \neq \emptyset$, and (ii) the empty set, of rank 0 .

We use only the simplest type of lift and truncation. The $i$-fold lift $L^{i}(M)$ of $M$ is $(M \times X) \backslash X$ where $|X|=i$; dually, $(M+X) / X$ is the $i$-fold truncation, $T^{i}(M)$.

The nullity of a set $Y$ is $\eta(Y)=|Y|-r(Y)$. Let $\mathcal{Z}^{\prime}(M)$ be the set of nonempty proper cyclic flats of $M$ and let $\eta\left(\mathcal{Z}^{\prime}(M)\right)$ be the sum of the nullities of these flats. The following lemma is easy to prove. 
Lemma 2.2. If $F \in \mathcal{Z}(M \backslash x)$, then $\operatorname{cl}_{M}(F) \in \mathcal{Z}(M)$. If $\operatorname{cl}_{M}(F)=F$, then $\eta_{M \backslash x}(F)$ is $\eta_{M}(F)$; if $\mathrm{cl}_{M}(F)=F \cup x$, then $\eta_{M \backslash x}(F)=\eta_{M}(F \cup x)-1$.

Dually, if $F \in \mathcal{Z}(M / y)$, then exactly one of $F$ and $F \cup y$ is in $\mathcal{Z}(M)$. The nullities of $F$ in $M / y$ and the corresponding cyclic flat of $M$ agree unless $y$ is a loop of $M$, in which case $\eta_{M / y}(F)=\eta_{M}(F \cup y)-1$.

Thus, if $N$ is a minor of $M$, then $\eta\left(\mathcal{Z}^{\prime}(N)\right) \leq \eta\left(\mathcal{Z}^{\prime}(M)\right)$.

While a cyclic flat of a matroid may give rise to cyclic flats in its restrictions, the next lemma identifies a situation in which this does not happen.

Lemma 2.3. Let $Z$ be a cyclic flat of $M$. If a subset $U$ of $Z$ with $|U| \geq \eta(Z)$ is contained in all nonempty cyclic flats that are contained in $Z$, then $Z-U$ is independent.

Proof. Assume, to the contrary, that $Z-U$ contains some circuit $C$. The nonempty cyclic flat $\operatorname{cl}(C)$ is contained in $Z$, so $U \subseteq \operatorname{cl}(C)$. Thus $U \subseteq \operatorname{cl}(Z-U)=Z$. Now $\eta(Z-U)>0$ and $U \subseteq \operatorname{cl}(Z-U)$ give $\eta(Z)>|U|$, contrary to the assumed inequality.

For matroids $M_{1}$ on $S_{1}$ and $M_{2}$ on $S_{2}$, a matroid $M$ on $S_{1} \cup S_{2}$ with $M \mid S_{1}=M_{1}$ and $M \mid S_{2}=M_{2}$ is called an amalgam of $M_{1}$ and $M_{2}$.

An element $x$ in a matroid $M$ is free in $M$ if $M=(M \backslash x)+x$. Dually, an element $y$ is cofree in $M$ if $M=(M / y) \times y$. Let $F I(M)$ be the set of all elements of $M$ that are in no proper cyclic flat of $M$; thus, $F I(M)$ consists of the free elements and isthmuses of $M$. Note that $F I\left(M^{*}\right)$ is the intersection of all nonempty cyclic flats of $M$; it consists of the cofree elements and loops of $M$.

\section{INTERTWINES}

We now construct the matroids of interest. The notation established in this paragraph is used in the rest of the paper. Assume the matroids $M_{1}$ and $M_{2}$ have positive rank and are defined on disjoint ground sets, $S_{1}$ and $S_{2}$, respectively. Let $r_{1}$ and $r_{2}$ be their rank functions, and let $\eta_{1}$ and $\eta_{2}$ be their nullity functions. Fix subsets $S_{1}^{\prime}$ of $S_{1}$ and $S_{2}^{\prime}$ of $S_{2}$, an integer $k$ with

$$
k \geq r\left(M_{1}\right)+\eta_{1}\left(S_{1}^{\prime}\right)+r\left(M_{2}\right)+\eta_{2}\left(S_{2}^{\prime}\right),
$$

and sets $T_{1}$ and $T_{2}$ with

$$
\left|T_{1}\right|=k-r\left(M_{1}\right)-\left|S_{2}^{\prime}\right| \quad \text { and } \quad\left|T_{2}\right|=k-r\left(M_{2}\right)-\left|S_{1}^{\prime}\right|
$$

where $T_{1}, T_{2}$, and $S_{1} \cup S_{2}$ are mutually disjoint. Let

$$
\mathcal{Z}=\mathcal{Z}^{\prime}\left(M_{1} \times\left(T_{1} \cup S_{2}^{\prime}\right)\right) \cup \mathcal{Z}^{\prime}\left(M_{2} \times\left(T_{2} \cup S_{1}^{\prime}\right)\right) \cup\left\{\emptyset, S_{1} \cup S_{2} \cup T_{1} \cup T_{2}\right\} .
$$

(Note that inequality (3.1) gives $\left|T_{1}\right|+\left|S_{2}^{\prime}\right| \geq \eta_{1}\left(S_{1}^{\prime}\right)+r\left(M_{2}\right)+\eta_{2}\left(S_{2}^{\prime}\right)$, which is positive; therefore $M_{1} \times\left(T_{1} \cup S_{2}^{\prime}\right)$ is a proper coextension of $M_{1}$ and so has no loops. Likewise $M_{2} \times\left(T_{2} \cup S_{1}^{\prime}\right)$ has no loops. Thus, the least cyclic flat of these matroids is $\emptyset$.) Define $r: \mathcal{Z} \rightarrow \mathbb{Z}$ by

(1) $r\left(F \cup T_{1} \cup S_{2}^{\prime}\right)=r_{1}(F)+\left|T_{1}\right|+\left|S_{2}^{\prime}\right|$ for $F \in \mathcal{Z}^{\prime}\left(M_{1}\right)$,

(2) $r\left(F \cup T_{2} \cup S_{1}^{\prime}\right)=r_{2}(F)+\left|T_{2}\right|+\left|S_{1}^{\prime}\right|$ for $F \in \mathcal{Z}^{\prime}\left(M_{2}\right)$,

(3) $r(\emptyset)=0$, and

(4) $r\left(S_{1} \cup S_{2} \cup T_{1} \cup T_{2}\right)=k$.

Theorem 3.1. The pair $(\mathcal{Z}, r)$ satisfies properties $(Z 0)-(Z 3)$ of Proposition 2.1 The rank- $k$ matroid $M$ on $S_{1} \cup S_{2} \cup T_{1} \cup T_{2}$ thus defined has the following properties:

(i) $M$ is an amalgam of $M_{1} \times\left(T_{1} \cup S_{2}^{\prime}\right)$ and $M_{2} \times\left(T_{2} \cup S_{1}^{\prime}\right)$, and 
(ii) $\eta_{1}(F)=\eta_{M}\left(F \cup T_{1} \cup S_{2}^{\prime}\right)$ for $F \in \mathcal{Z}^{\prime}\left(M_{1}\right)$ and $\eta_{2}(F)=\eta_{M}\left(F \cup T_{2} \cup S_{1}^{\prime}\right)$ for $F \in \mathcal{Z}^{\prime}\left(M_{2}\right)$.

Proof. Property (Z0) holds since any pair of sets in $\mathcal{Z}$ that does not have a join in one of $\mathcal{Z}^{\prime}\left(M_{1} \times\left(T_{1} \cup S_{2}^{\prime}\right)\right)$ and $\mathcal{Z}^{\prime}\left(M_{2} \times\left(T_{2} \cup S_{1}^{\prime}\right)\right)$ has $S_{1} \cup S_{2} \cup T_{1} \cup T_{2}$ as the join. Property (Z1) is item (3) above. Property (Z2) follows from this property in $\mathcal{Z}\left(M_{1} \times\left(T_{1} \cup S_{2}^{\prime}\right)\right)$ and $\mathcal{Z}\left(M_{2} \times\left(T_{2} \cup S_{1}^{\prime}\right)\right)$, as do all instances of property (Z3) except in the case $X=F_{1} \cup T_{1} \cup S_{2}^{\prime}$ with $F_{1} \in \mathcal{Z}\left(M_{1}\right)$ and $Y=F_{2} \cup T_{2} \cup S_{1}^{\prime}$ with $F_{2} \in \mathcal{Z}\left(M_{2}\right)$. In this case, the required inequality is

$$
r_{1}\left(F_{1}\right)+\left|T_{1}\right|+\left|S_{2}^{\prime}\right|+r_{2}\left(F_{2}\right)+\left|T_{2}\right|+\left|S_{1}^{\prime}\right| \geq k+\left|F_{1} \cap S_{1}^{\prime}\right|+\left|F_{2} \cap S_{2}^{\prime}\right|,
$$

which follows from inequality (3.1) and equations (3.2).

By symmetry, assertion (i) follows if we show that for any $F \in \mathcal{Z}^{\prime}\left(M_{2}\right)$, the difference $\left(F \cup T_{2} \cup S_{1}^{\prime}\right)-\left(T_{2} \cup\left(S_{2}-S_{2}^{\prime}\right)\right)$ is independent in $M \mid S_{1} \cup T_{1} \cup S_{2}^{\prime}$. All such differences are contained in $S_{1}^{\prime} \cup S_{2}^{\prime}$, so it suffices to show that $S_{1}^{\prime} \cup S_{2}^{\prime}$ is independent in $M$. To show this, by equation (2.1) it suffices to prove

$$
r_{M}(Z)+\left|\left(S_{1}^{\prime} \cup S_{2}^{\prime}\right)-Z\right| \geq\left|S_{1}^{\prime}\right|+\left|S_{2}^{\prime}\right|
$$

for all $Z \in \mathcal{Z}(M)$; again by symmetry, it suffices to show this for $Z=F_{1} \cup T_{1} \cup S_{2}^{\prime}$ with $F_{1} \in \mathcal{Z}^{\prime}\left(M_{1}\right)$. For such $Z$, the required inequality is

$$
r_{1}\left(F_{1}\right)+\left|T_{1}\right|+\left|S_{2}^{\prime}\right|+\left|S_{1}^{\prime}-F_{1}\right| \geq\left|S_{1}^{\prime}\right|+\left|S_{2}^{\prime}\right|,
$$

or, using equations (3.2) and manipulating,

$$
k \geq r\left(M_{1}\right)+\left|S_{1}^{\prime} \cap F_{1}\right|-r_{1}\left(F_{1}\right)+\left|S_{2}^{\prime}\right| .
$$

This inequality follows from inequality (3.1) since $\left|S_{2}^{\prime}\right| \leq r\left(M_{2}\right)+\eta_{2}\left(S_{2}^{\prime}\right)$ and

$$
\begin{aligned}
\left|S_{1}^{\prime} \cap F_{1}\right|-r_{1}\left(F_{1}\right) & \leq\left|S_{1}^{\prime} \cap F_{1}\right|-r_{1}\left(S_{1}^{\prime} \cap F_{1}\right) \\
& =\eta_{1}\left(S_{1}^{\prime} \cap F_{1}\right) \\
& \leq \eta_{1}\left(S_{1}^{\prime}\right) .
\end{aligned}
$$

Assertion (ii) is evident.

The matroid so constructed depends on $M_{1}, M_{2}, k, S_{1}^{\prime}, S_{2}^{\prime}, T_{1}$, and $T_{2}$. If (as in the next result) listing all parameters aids clarity, we use $M_{k}\left(M_{1}, S_{1}^{\prime}, T_{1} ; M_{2}, S_{2}^{\prime}, T_{2}\right)$ to denote this matroid; otherwise we simply write $M$.

The next result, which follows by comparing the cyclic flats and their ranks, shows that combining the construction with duality yields other instances of the same construction.

Theorem 3.2. With $j=\left|S_{1}\right|+\left|S_{2}\right|+\left|T_{1}\right|+\left|T_{2}\right|-k$, we have

$$
\left(M_{k}\left(M_{1}, S_{1}^{\prime}, T_{1} ; M_{2}, S_{2}^{\prime}, T_{2}\right)\right)^{*}=M_{j}\left(M_{1}^{*}, S_{1}-S_{1}^{\prime}, T_{2} ; M_{2}^{*}, S_{2}-S_{2}^{\prime}, T_{1}\right) .
$$

Also, $j \geq r\left(M_{1}^{*}\right)+\eta_{M_{1}^{*}}\left(S_{1}-S_{1}^{\prime}\right)+r\left(M_{2}^{*}\right)+\eta_{M_{2}^{*}}\left(S_{2}-S_{2}^{\prime}\right)$ if and only if $k$ satisfies inequality (3.1).

We now treat the main result. A similar but somewhat longer argument would modestly increase the range for $k$; we opt for the shorter proof since the main interest is in having infinitely many intertwines. Recall that $F I(M)$ is the set of free elements and isthmuses of $M$, so $F I\left(M^{*}\right)$ is the set of cofree elements and loops of $M$. 
Theorem 3.3. Assume that the ground sets $S_{1}$ and $S_{2}$ of $M_{1}$ and $M_{2}$ are disjoint and that no matroid isomorphic to $M_{1}$ (resp., $M_{2}$ ) can be obtained from $M_{2}$ (resp., $M_{1}$ ) by any combination of minors, free extensions, and free coextensions. For $i \in\{1,2\}$, fix a set $S_{i}^{\prime}$ with $F I\left(M_{i}\right) \subseteq S_{i}^{\prime} \subseteq S_{i}-F I\left(M_{i}^{*}\right)$. If $k \geq 4 \max \left\{\left|S_{1}\right|,\left|S_{2}\right|\right\}$, then the matroid $M$ defined above is an intertwine of $M_{1}$ and $M_{2}$.

Proof. Theorem 3.1 part (i) shows that $M_{1}$ and $M_{2}$ are minors of $M$. By symmetry, to prove that $M$ is an intertwine, it suffices to show that for $a \in S_{1} \cup T_{1}$, neither $M \backslash a$ nor $M / a$ has both an $M_{1}$-minor and an $M_{2}$-minor; furthermore, by Theorem 3.2 and the observation that the hypotheses are invariant under duality, it suffices to treat $M \backslash a$. Now $\left|T_{1}\right| \geq r\left(M_{1}^{*}\right)+r\left(M_{2}\right)+\left|S_{i}\right|+1$ since $k \geq 4 \max \left\{\left|S_{1}\right|,\left|S_{2}\right|\right\}$. If $M \backslash a \backslash X / Y \simeq M_{i}$ with $i \in\{1,2\}$, then $M \backslash a \backslash X / Y$ has $\left|S_{i}\right|$ elements, at least $\left|T_{1}\right|-\left|X \cap T_{1}\right|-\left|Y \cap T_{1}\right|-1$ of which are in $T_{1}$, so $\left|X \cap T_{1}\right|+\left|Y \cap T_{1}\right| \geq\left|T_{1}\right|-\left|S_{i}\right|-1$, and therefore

$$
\left|X \cap T_{1}\right|+\left|Y \cap T_{1}\right| \geq r\left(M_{1}^{*}\right)+r\left(M_{2}\right) \text {. }
$$

Thus, either (i) $\left|X \cap T_{1}\right| \geq r\left(M_{1}^{*}\right)$ or (i*) $\left|Y \cap T_{1}\right| \geq r\left(M_{2}\right)$.

We claim that the three conclusions below follow when inequality (i) holds:

(1) $M \backslash\left(X \cap T_{1}\right)=\left(M_{2} \times\left(T_{2} \cup S_{1}^{\prime}\right)\right)+\left(\left(S_{1}-S_{1}^{\prime}\right) \cup\left(T_{1}-X\right)\right)$,

(2) $M \backslash\left(X \cap T_{1}\right)$ has no $M_{1}$-minor, and

(3) $\eta_{2}(F)=\eta_{M \backslash\left(X \cap T_{1}\right)}\left(F \cup T_{2} \cup S_{1}^{\prime}\right)$ for $F \in \mathcal{Z}^{\prime}\left(M_{2}\right)$.

Item (1) holds since, using Lemma 2.3, we get that the proper cyclic flats and their ranks in the two matroids agree. Item (1) and the hypotheses give item (2). Item (3) is immediate.

Inequalities (i) and $\left(i^{*}\right)$ are related by duality, so Theorem 3.2 and the results in the last paragraph give the following conclusion if inequality $\left(\mathrm{i}^{*}\right)$ holds:

$\left(1^{*}\right) M /\left(Y \cap T_{1}\right)=\left(M_{1} \times\left(\left(T_{1}-Y\right) \cup S_{2}^{\prime}\right)\right)+\left(\left(S_{2}-S_{2}^{\prime}\right) \cup T_{2}\right)$,

(2*) $M /\left(Y \cap T_{1}\right)$ has no $M_{2}$-minor, and

(3*) $\eta_{1}(F)=\eta_{M /\left(Y \cap T_{1}\right)}\left(F \cup\left(T_{1}-Y\right) \cup S_{2}^{\prime}\right)$ for $F \in \mathcal{Z}^{\prime}\left(M_{1}\right)$.

For $a \in\left(S_{1}-S_{1}^{\prime}\right) \cup T_{1}$, assume $M \backslash a$ has an $M_{1}$-minor, say $M \backslash a \backslash X / Y$. By item (2), inequality (i*) holds. Since $a$ is in at least one set in $\mathcal{Z}^{\prime}\left(M /\left(Y \cap T_{1}\right)\right)$, item $\left(3^{*}\right)$ gives $\eta\left(\mathcal{Z}^{\prime}\left(M /\left(Y \cap T_{1}\right) \backslash a\right)\right)<\eta\left(\mathcal{Z}^{\prime}\left(M_{1}\right)\right)$; the contradiction $M \backslash a \backslash X / Y \nsucceq M_{1}$ now follows from Lemma 2.2

Lastly, for $a \in S_{1}^{\prime}$, assume $M \backslash a$ has an $M_{2}$-minor, say $M \backslash a \backslash X / Y$. Inequality (i) holds by item $\left(2^{*}\right)$. Now $a \in S_{1}^{\prime}$ gives $\eta\left(\mathcal{Z}^{\prime}\left(M \backslash\left(X \cap T_{1}\right) \backslash a\right)\right)<\eta\left(\mathcal{Z}^{\prime}\left(M_{2}\right)\right)$, which, with Lemma2.2. gives the contradiction $M \backslash a \backslash X / Y \nsucceq M_{2}$.

Assume $F I\left(M_{i}\right)=\emptyset=F I\left(M_{i}^{*}\right)$ for $i \in\{1,2\}$. Reflecting on the proof above shows that $a \in S_{1}$ if and only if neither $M \backslash a$ nor $M / a$ has an $M_{1}$-minor, and likewise for $S_{2}$ and $M_{2}$. These conclusions and the structure of the cyclic flats of $M$ show that the counterparts of the sets $S_{1}, S_{2}, T_{1}, T_{2}, S_{1}^{\prime}$, and $S_{2}^{\prime}$ can be determined from any matroid that is isomorphic to $M$. This gives the following result.

Corollary 3.4. Assume $F I\left(M_{i}\right)=\emptyset=F I\left(M_{i}^{*}\right)$ for $i \in\{1,2\}$. The construction gives at least $\left(\left|S_{1}\right|+1\right)\left(\left|S_{2}\right|+1\right)$ nonisomorphic rank- $k$ intertwines of $M_{1}$ and $M_{2}$ for each integer $k \geq 4 \max \left\{\left|S_{1}\right|,\left|S_{2}\right|\right\}$. If, in addition, both $M_{1}$ and $M_{2}$ have trivial automorphism groups, then the construction yields $2^{\left|S_{1}\right|+\left|S_{2}\right|}$ nonisomorphic rank- $k$ intertwines.

Knowing more about $M_{1}$ and $M_{2}$ may suggest variations on the construction that yield more intertwines, as we now illustrate. Assume that in addition to satisfying the conditions in Theorem 3.3, neither $M_{1}$ nor $M_{2}$ has circuit-hyperplanes. Let $M$ be the intertwine constructed above. From the bound on $k$ in Theorem 3.3 we get $\left|T_{1} \cup T_{2}\right|>k$. Let $\mathcal{H}$ 
be a collection of $k$-subsets of $T_{1} \cup T_{2}$ with $\left|H \cap H^{\prime}\right| \leq k-2$ whenever $H$ and $H^{\prime}$ are distinct sets in $\mathcal{H}$. In the construction, replace $\mathcal{Z}$ by $\mathcal{Z} \cup \mathcal{H}$ and extend $r$ to $\mathcal{Z} \cup \mathcal{H}$ by setting $r(H)=k-1$ for all $H \in \mathcal{H}$. Properties (Z0)-(Z3) of Proposition 2.1 are easily verified. Let $M^{\prime}$ be the matroid thus constructed. The sets in $\mathcal{H}$ are the circuit-hyperplanes of $M^{\prime}$. By comparing the cyclic flats and their ranks, it follows that if $M^{\prime} \backslash X / Y$ has no circuit-hyperplanes, then $M^{\prime} \backslash X / Y=M \backslash X / Y$. Since neither $M_{1}$ nor $M_{2}$ has circuithyperplanes, it follows that if some single-element deletion or contraction of $M^{\prime}$ had both an $M_{1}$-minor and an $M_{2}$-minor, then the same would be true of the corresponding singleelement deletion or contraction of $M$, contrary to Theorem 3.3. Thus, $M^{\prime}$ is an intertwine of $M_{1}$ and $M_{2}$. Thus, we have the following result.

Theorem 3.5. Assume $M_{1}$ and $M_{2}$ satisfy the hypotheses of Theorem 3.3 and neither has circuit-hyperplanes. For each integer $n$, there is an integer $k_{0}$ so that if $k \geq k_{0}$, then $M_{1}$ and $M_{2}$ have at least $n$ intertwines of rank $k$.

To take these ideas a step further, we give a simple proof that, as $k$ grows, the number of nonisomorphic intertwines arising from the variation on the construction grows at least exponentially. To simplify the discussion slightly, assume both $\left|T_{1} \cup T_{2}\right|$ and $k$ are even. Let $\mathscr{H}$ be the set of all sets $\mathcal{H}$ of $k$-subsets of $T_{1} \cup T_{2}$ such that $\left|H \cap H^{\prime}\right| \leq k-2$ whenever $H$ and $H^{\prime}$ are distinct sets in $\mathcal{H}$. One way to get a set $\mathcal{H}$ in $\mathscr{H}$ is to pair off the elements in $T_{1} \cup T_{2}$ and, to get each set in $\mathcal{H}$, choose $k / 2$ pairs. Even among sets $\mathcal{H}$ formed in this limited way, their maximal size grows exponentially as a function of $k$ (much as $\left(\begin{array}{c}2 n \\ n\end{array}\right)$ grows exponentially as a function of $n$ ). Among all sets in $\mathscr{H}$, let $\mathcal{H}$ be one of maximal size. Subsets of $\mathcal{H}$ of different sizes give rise to nonisomorphic intertwines (their numbers of circuit-hyperplanes differ), so these intertwines demonstrate our claim.

This discussion and the last two results suggest several problems. Let $i\left(k ; M_{1}, M_{2}\right)$ denote the number of rank- $k$ intertwines of $M_{1}$ and $M_{2}$ up to isomorphism. What can be said about $i\left(k ; M_{1}, M_{2}\right)$ ? If $M_{1}$ and $M_{2}$ satisfy the hypotheses of Theorem 3.3, is $i\left(k ; M_{1}, M_{2}\right)$ increasing as a function of $k$, at least for sufficiently large $k$ ? If so, under what conditions on $M_{1}$ and $M_{2}$ is the difference $i\left(k+1 ; M_{1}, M_{2}\right)-i\left(k ; M_{1}, M_{2}\right)$ bounded above by a constant or by a polynomial? Under what conditions does $i\left(k ; M_{1}, M_{2}\right)$ grow exponentially or super-exponentially?

A matroid $M$ is a labelled intertwine of $M_{1}$ and $M_{2}$ if $M$ but none of its proper minors has minors equal to $M_{1}$ and $M_{2}$. We end this section by showing that weaker hypotheses than those in Theorem 3.3 suffice for our construction to yield labelled intertwines.

Theorem 3.6. Assume $S_{1}$ and $S_{2}$ are disjoint. If inequality (3.1) holds, neither $M_{1}$ nor $M_{2}$ is uniform, $\mathcal{Z}^{\prime}\left(M_{1}\right) \neq\left\{S_{1}^{\prime}\right\}$, and $\mathcal{Z}^{\prime}\left(M_{2}\right) \neq\left\{S_{2}^{\prime}\right\}$, then the matroid $M$ constructed above is a labelled intertwine of $M_{1}$ and $M_{2}$.

Proof. By symmetry, to prove that no proper minor of $M$ has both $M_{1}$ and $M_{2}$ as minors, it suffices to show that if $M \backslash X / Y=M_{1}$, then $X=\left(S_{2}-S_{2}^{\prime}\right) \cup T_{2}$ and $Y=T_{1} \cup S_{2}^{\prime}$. Thus, assume $M \backslash X / Y=M_{1}$. Fix $F \in \mathcal{Z}^{\prime}\left(M_{1}\right)-\left\{S_{1}^{\prime}\right\}$. By Lemma 2.2, the cyclic flat $F$ of $M \backslash X / Y$ must arise from the cyclic flat $F \cup T_{1} \cup S_{2}^{\prime}$ of $M$; from Theorem 3.1 part (ii), it follows that for $M \backslash X / Y$ to yield the same nullity on $F$ as in $M_{1}$, each element of $T_{1} \cup S_{2}^{\prime}$ must be contracted; dually, each element of $\left(S_{2}-S_{2}^{\prime}\right) \cup T_{2}$ must be deleted. Thus, $X=\left(S_{2}-S_{2}^{\prime}\right) \cup T_{2}$ and $Y=T_{1} \cup S_{2}^{\prime}$.

\section{FURTHER RESUlts}

Among the pairs of matroids that Theorem 3.3 applies to are any two spikes of rank at least 4 , neither of which is a minor of the other, provided that the one of smaller rank (if the 
ranks differ) is not a free spike. (We use the definition of spikes in [3], which some sources call tip-less spikes. Free spikes are the only spikes that can be obtained from a spike by minors along with at least one lift or truncation.) Thus, the assumption in the construction in [3] that each element is in a dependent transversal is not needed here. However, unlike the construction in [3], the intertwine we get when $M_{1}$ and $M_{2}$ are spikes is not a spike.

The construction here and that in [3] give intertwines with contrasting properties and so show that some properties that hold for one construction need not hold for intertwines in general. For instance, the intertwines constructed here have neither small circuits nor small cocircuits, but those constructed in [3] have each element in a many 4-circuits and in many 4-cocircuits. Also, in our construction the number of cyclic flats does not depend on the rank, but in the construction in [3] the number of cyclic flats grows with the rank (as is true for the variation we discussed before Theorem 3.5).

4.1. Sizes of intertwines. We show that the intertwines constructed above can exhibit the full range of possible sizes for each rank.

Theorem 4.1. If $S$ is the ground set of a rank-k intertwine of $M_{1}$ and $M_{2}$, then

$$
2 k-r\left(M_{1}\right)-r\left(M_{2}\right) \leq|S| \leq 2 k+r\left(M_{1}^{*}\right)+r\left(M_{2}^{*}\right) .
$$

If $F I\left(M_{i}\right)=\emptyset=F I\left(M_{i}^{*}\right)$ for $i \in\{1,2\}$, then the construction in Section 3 gives intertwines of each cardinality in this range.

Proof. Let $M$, on the set $S$, be a rank- $k$ intertwine of $M_{1}$ and $M_{2}$, so $M \backslash X / Y \simeq M_{1}$ and $M \backslash X^{\prime} / Y^{\prime} \simeq M_{2}$ for some subsets $X, Y, X^{\prime}, Y^{\prime}$ of $S$. Standard arguments about minors show that we may assume that $Y$ and $Y^{\prime}$ are independent sets with $|Y|=k-r\left(M_{1}\right)$ and $\left|Y^{\prime}\right|=k-r\left(M_{2}\right)$. No proper contraction of $M$ has both $M_{1}$ - and $M_{2}$-minors, so $Y \cap Y^{\prime}=\emptyset$; thus, $|Y|+\left|Y^{\prime}\right| \leq|S|$, so $k-r\left(M_{1}\right)+k-r\left(M_{2}\right) \leq|S|$. This lower bound on $|S|$ is achieved in the construction when $S_{1}^{\prime}=S_{1}$ and $S_{2}^{\prime}=S_{2}$. No proper deletion of $M$ has both $M_{1}$ - and $M_{2}$-minors, so $X \cap X^{\prime}=\emptyset$; thus, $|X| \leq\left|S_{2}\right|+\left|Y^{\prime}\right|$. This inequality, the equation $|S|=\left|S_{1}\right|+|X|+|Y|$, and values for $|Y|$ and $\left|Y^{\prime}\right|$ give the upper bound. This bound in attained when $S_{1}^{\prime}=\emptyset=S_{2}^{\prime}$. By varying $\left|S_{1}^{\prime}\right|$ and $\left|S_{2}^{\prime}\right|$, all cardinalities between these bounds can be realized.

4.2. Representable matroids. All spikes are contained in $\mathcal{E}\left(U_{2,6}, U_{4,6}\right)$, the class of matroids that have neither $U_{2,6^{-}}$nor $U_{4,6}$-minors. The results in this subsection and the next are akin to a corollary that Vertigan got from his work on intertwines and spikes: some pairs of matroids in $\mathcal{E}\left(U_{2,6}, U_{4,6}\right)$ have infinitely many intertwines in $\mathcal{E}\left(U_{2,6}, U_{4,6}\right)$.

The result below uses the following equivalent formulations of two special cases of our construction. (The first assertion follows by comparing the cyclic flats and their ranks; the second is the dual of the first. Recall that $T^{k}$ and $L^{j}$ denote truncations and lifts.) If $k \geq r\left(M_{1}\right)+r\left(M_{2}\right)$, then

$$
M_{k}\left(M_{1}, \emptyset, T_{1} ; M_{2}, \emptyset, T_{2}\right)=T^{k}\left(\left(M_{1} \times T_{1}\right) \oplus\left(M_{2} \times T_{2}\right)\right) .
$$

If $k \geq r\left(M_{1}\right)+\left|S_{1}\right|+r\left(M_{2}\right)+\left|S_{2}\right|$ and $j=k-r\left(M_{1}\right)-r\left(M_{2}\right)$, then

$$
M_{k}\left(M_{1}, S_{1}, T_{1} ; M_{2}, S_{2}, T_{2}\right)=L^{j}\left(\left(M_{1}+T_{2}\right) \oplus\left(M_{2}+T_{1}\right)\right) .
$$

Corollary 4.2. Assume a class $\mathcal{C}$ of matroids is closed under direct sum, free extension, free coextension, truncation, and lift. If $M_{1}, M_{2} \in \mathcal{C}$ satisfy the hypotheses of Theorem 3.3 and if either $F I\left(M_{1}\right)=\emptyset=F I\left(M_{2}\right)$ or $F I\left(M_{1}^{*}\right)=\emptyset=F I\left(M_{2}^{*}\right)$, then $\mathcal{C}$ contains infinitely many intertwines of $M_{1}$ and $M_{2}$. 
Such classes $\mathcal{C}$ include the class of matroids that are representable over a given infinite field and the class of matroids that are representable over a given characteristic.

4.3. Transversal matroids and gammoids. We next show that the intertwine $M$ that we constructed is transversal if and only if $M_{1}$ and $M_{2}$ are; we also treat the corresponding statements for several related types of matroids. We will use the characterization of transversal matroids in Lemma 4.3, which is due to Ingleton [4] and refines a result of Mason. For a collection $\mathcal{F}$ of sets, let $\cap \mathcal{F}$ be $\bigcap_{X \in \mathcal{F}} X$ and $\cup \mathcal{F}$ be $\bigcup_{X \in \mathcal{F}} X$.

Lemma 4.3. A matroid $M$ is transversal if and only if for all $\mathcal{A} \subseteq \mathcal{Z}(M)$ with $\mathcal{A} \neq \emptyset$,

$$
\sum_{\mathcal{F} \subseteq \mathcal{A}}(-1)^{|\mathcal{F}|+1} r(\cup \mathcal{F}) \geq r(\cap \mathcal{A})
$$

In this result, it suffices to consider only antichains $\mathcal{A}$ of cyclic flats since if $X, Y \in \mathcal{A}$ with $X \subset Y$, then using $\mathcal{A}-\{Y\}$ in place of $\mathcal{A}$ does not change either side of inequality (4.1); with $\mathcal{A}$, the terms on the left side that include $Y$ cancel via the involution that adjoins $X$ to, or omits $X$ from, $\mathcal{F}$. Also, it suffices to focus on inequality (4.1) for $|\mathcal{A}|>2$ since equality holds when $|\mathcal{A}|=1$ and the case of $|\mathcal{A}|=2$ is the semimodular inequality.

Corollary 4.4. A matroid $M$ is transversal if and only if $M \times x$ is.

Cotransversal matroids are duals of transversal matroids. Bitransversal matroids are both transversal and cotransversal. Gammoids are minors of transversal matroids. Restrictions of transversal matroids are transversal, so any gammoid is a contraction of some transversal matroid; it follows that any gammoid is a nullity-preserving contraction of some transversal matroid. The class of gammoids is closed under duality, so any gammoid has a rank-preserving extension to a cotransversal matroid.

Theorem 4.5. Assume inequality (3.1) holds. The matroids $M_{1}$ and $M_{2}$ are transversal if and only if $M=M_{k}\left(M_{1}, S_{1}^{\prime}, T_{1} ; M_{2}, S_{2}^{\prime}, T_{2}\right)$ is. The corresponding statements hold for cotransversal matroids, bitransversal matroids, and gammoids.

Proof. Since $M_{1} \times\left(T_{1} \cup S_{2}^{\prime}\right)$ and $M_{2} \times\left(T_{2} \cup S_{1}^{\prime}\right)$ are restrictions of $M$, from Corollary 4.4 it follows that if $M$ is transversal, then so are $M_{1}$ and $M_{2}$. Now assume $M_{1}$ and $M_{2}$ are transversal. Let $\mathcal{A}$ be an antichain in $\mathcal{Z}(M)$ with $|\mathcal{A}|>2$. Set

$$
\mathcal{A}_{1}=\mathcal{A} \cap \mathcal{Z}^{\prime}\left(M_{1} \times\left(T_{1} \cup S_{2}^{\prime}\right)\right) \quad \text { and } \quad \mathcal{A}_{2}=\mathcal{A} \cap \mathcal{Z}^{\prime}\left(M_{2} \times\left(T_{2} \cup S_{1}^{\prime}\right)\right) .
$$

Thus, $\mathcal{A}$ is the disjoint union of $\mathcal{A}_{1}$ and $\mathcal{A}_{2}$. By Corollary 4.4 and Lemma 4.3 inequality (4.1) holds for $\mathcal{A}_{1}$ if it is nonempty, and likewise for $\mathcal{A}_{2}$; thus, this inequality holds for $\mathcal{A}$ if one of $\mathcal{A}_{1}$ and $\mathcal{A}_{2}$ is empty. Assume neither is empty. For $F_{1} \in \mathcal{A}_{1}$ and $F_{2} \in \mathcal{A}_{2}$, we have $r_{M}\left(F_{1} \cup F_{2}\right)=r(M)$, so

$$
\begin{aligned}
\sum_{\mathcal{F} \subseteq \mathcal{A}}(-1)^{|\mathcal{F}|+1} r(\cup \mathcal{F})= & \sum_{\mathcal{F}_{1} \subseteq \mathcal{A}_{1}}(-1)^{\left|\mathcal{F}_{1}\right|+1} r\left(\cup \mathcal{F}_{1}\right)+\sum_{\substack{\mathcal{F}_{2} \subseteq \mathcal{A}_{2}\\
}}(-1)^{\left|\mathcal{F}_{2}\right|+1} r\left(\cup \mathcal{F}_{2}\right) \\
& \quad \sum_{\substack{\mathcal{F}_{1} \subseteq \mathcal{A}_{1}, \mathcal{F}_{1} \neq \emptyset \\
\mathcal{F}_{2} \subseteq \mathcal{A}_{2}, \mathcal{F}_{2} \neq \emptyset}}(-1)^{\left|\mathcal{F}_{1}\right|+\left|\mathcal{F}_{2}\right|+1} r(M) \\
\geq & r\left(\cap \mathcal{A}_{1}\right)+r\left(\cap \mathcal{A}_{2}\right)-r(M) \\
\geq & r(\cap \mathcal{A}),
\end{aligned}
$$

where the last line follows from semimodularity along with the inclusions $T_{1} \cup S_{2}^{\prime} \subseteq \cap \mathcal{A}_{1}$ and $T_{2} \cup S_{1}^{\prime} \subseteq \cap \mathcal{A}_{2}$, and the fact that $T_{1} \cup T_{2} \cup S_{1}^{\prime} \cup S_{2}^{\prime}$ spans $M$ (a consequence of equation (2.1) and inequality (3.1)). Thus, inequality (4.1) holds, so $M$ is transversal. 
The assertions about cotransversal and bitransversal matroids follow by Theorem 3.2 .

If $M$ is a gammoid, then so are its minors $M_{1}$ and $M_{2}$. Now assume $M_{1}$ and $M_{2}$ are gammoids. Let $M_{1}^{\prime}$ and $M_{2}^{\prime}$ be rank-preserving cotransversal extensions of $M_{1}$ and $M_{2}$. Thus, $M_{k}\left(M_{1}^{\prime}, S_{1}^{\prime}, T_{1} ; M_{2}^{\prime}, S_{2}^{\prime}, T_{2}\right)$ is cotransversal since inequality (3.1) holds with $M_{1}^{\prime}$ and $M_{2}^{\prime}$ in place of $M_{1}$ and $M_{2}$. Comparing the cyclic flats and their ranks shows that $M$ is a restriction of $M_{k}\left(M_{1}^{\prime}, S_{1}^{\prime}, T_{1} ; M_{2}^{\prime}, S_{2}^{\prime}, T_{2}\right)$, so $M$ is a gammoid.

Corollary 4.6. If $M_{1}$ and $M_{2}$ satisfy the hypotheses of Theorem 3.3 and are transversal, then infinitely many intertwines of $M_{1}$ and $M_{2}$ are transversal. The corresponding statements hold for cotransversal matroids, bitransversal matroids, and gammoids.

4.4. Uniform minors. We claim that $M \mid B_{1} \cup B_{2} \cup T_{1} \cup T_{2}$, where $B_{i}$ is a basis of $M_{i}$, is the

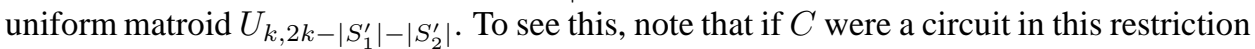
with $r(C)<k$, then $\mathrm{cl}_{M}(C) \in \mathcal{Z}^{\prime}(M)$; however, it follows from the construction that flats in $\mathcal{Z}^{\prime}(M)$ intersect $B_{1} \cup B_{2} \cup T_{1} \cup T_{2}$ in independent sets.

Corollary 4.7. If $M_{1}$ and $M_{2}$ satisfy the hypotheses of Theorem 3.3 then for any integer $n$, some intertwine of $M_{1}$ and $M_{2}$ has a $U_{n, 2 n}$-minor.

4.5. Connectivity. Recall that for any non-uniform matroid, $\lambda(M) \leq \kappa(M)$ where $\lambda(M)$ is the (Tutte) connectivity of $M$ and $\kappa(M)$ is its vertical connectivity. Thus, showing that the connectivity of intertwines can be arbitrarily large gives the counterpart for vertical connectivity.

Qin [6] proved $\lambda((M+p) \times q)-\lambda(M) \in\{1,2\}$ for any matroid $M$. For the matroid $M$ constructed above, fix a subset $T_{2}^{\prime}$ of $T_{2}$ with $\left|T_{2}^{\prime}\right|=\eta\left(M_{2}\right)$. Comparing the cyclic flats and their ranks, with the help of Lemma 2.3 , gives

$$
M \backslash T_{2}^{\prime}=\left(M_{1} \times\left(T_{1} \cup S_{2}^{\prime}\right)\right)+\left(\left(T_{2}-T_{2}^{\prime}\right) \cup\left(S_{2}-S_{2}^{\prime}\right)\right) .
$$

After some number of free coextensions or free extensions of $M_{1}$ according to the difference between $\left|T_{1} \cup S_{2}^{\prime}\right|$ and $\left|\left(T_{2}-T_{2}^{\prime}\right) \cup\left(S_{2}-S_{2}^{\prime}\right)\right|$ (which does not change as $k$ increases), the deletion $M \backslash T_{2}^{\prime}$ can be seen as resulting from free extension/free coextension pairs, so the connectivity of such deletions $M \backslash T_{2}^{\prime}$ grows with $k$. Since extending as needed by the elements in $T_{2}^{\prime}$ to obtain $M$ preserves the rank and introduces no circuits of size $\left|T_{2}\right|+\left|S_{1}^{\prime}\right|$ or smaller, $\lambda(M)$ also grows with $k$.

Corollary 4.8. If $M_{1}$ and $M_{2}$ satisfy the hypotheses of Theorem 3.3 then for any integer $n$, some intertwine of $M_{1}$ and $M_{2}$ is n-connected.

With the truncation that cuts the rank of the direct sum in half, it follows that the intertwine $T^{k}\left(\left(M_{1} \times T_{1}\right) \oplus\left(M_{2} \times T_{2}\right)\right)$ (arising from $\left.S_{1}^{\prime}=\emptyset=S_{2}^{\prime}\right)$ is rounded, that is, the ground set is not the union of two proper flats, or, equivalently, each cocircuit spans. (This notion, also called non-splitting, is equivalent to having $\kappa(M)=r(M)$.)

Note that in a rank- $n$ spike $M$ with $n \geq 4$, if $H$ is a hyperplane spanned by $n-2$ legs (using the terminology of [3]), then $(H, E(M)-H)$ is a vertical 3-separation of $M$. Thus, the construction in this paper and that in [3] yield intertwines with contrasting connectivity and vertical connectivity properties.

\section{The Relation to Vertigan's CONSTRUCtion}

As mentioned in the introduction, the first construction of infinite sets of intertwines for pairs of matroids was given by Dirk Vertigan. In this section we briefly outline his construction and show that, although the approaches differ, some instances of the two 
constructions coincide; furthermore, both approaches can be extended to yield the same collections of intertwines. Vertigan's theorem is as follows.

Theorem 5.1. Assume neither $M_{1}$ nor $M_{2}$ can be obtained, up to isomorphism, from the other by any combination of minors, free extensions, and free coextensions. If $F I\left(M_{i}\right)=$ $\emptyset=F I\left(M_{i}^{*}\right)$ for $i \in\{1,2\}$, then $M_{1}$ and $M_{2}$ have infinitely many intertwines.

The intertwines he constructed to prove this result are defined as follows. Let $S_{1}$ and $S_{2}$ be the ground sets of $M_{1}$ and $M_{2}$, which, in contrast to Theorem 3.3, need not be disjoint. Let $X$ and $Y$ be disjoint $k$-element sets, where $k \geq 10 \max \left\{\left|S_{1}\right|,\left|S_{2}\right|\right\}$, such that (i) $S_{1} \cup S_{2} \subseteq X \cup Y$, (ii) $X \cap S_{1}$ has $r\left(M_{1}\right)$ elements and is dependent in $M_{1}$, and (iii) $Y \cap S_{2}$ has $r\left(M_{2}\right)$ elements and is dependent in $M_{2}$. Set

$$
M_{1}^{\prime}=\left(M_{1}+\left(Y-S_{1}\right)\right) \times\left(X-S_{1}\right) \quad \text { and } \quad M_{2}^{\prime}=\left(M_{2}+\left(X-S_{2}\right)\right) \times\left(Y-S_{2}\right) \text {. }
$$

Thus, $r\left(M_{1}^{\prime}\right)=k=r\left(M_{2}^{\prime}\right)$. He argues that the intersection of the collections of bases of $M_{1}^{\prime}$ and $M_{2}^{\prime}$ is the collection of bases of a matroid on $X \cup Y$, and that this matroid is an intertwine of $M_{1}$ and $M_{2}$. Thus, this intertwine has rank $k$ and has $2 k$ elements. Vertigan observed that, as in Corollary 4.7, these intertwines have uniform minors of large rank and corank.

To relate this construction to ours, we first show that the bases of the intertwines we constructed can be described in a similar manner. Using the notation in Section 3 , set

$$
M_{1}^{\prime \prime}=\left(M_{1} \times\left(T_{1} \cup S_{2}^{\prime}\right)\right)+\left(T_{2} \cup\left(S_{2}-S_{2}^{\prime}\right)\right)
$$

and

$$
M_{2}^{\prime \prime}=\left(M_{2} \times\left(T_{2} \cup S_{1}^{\prime}\right)\right)+\left(T_{1} \cup\left(S_{1}-S_{1}^{\prime}\right)\right) .
$$

Both $M_{1}^{\prime \prime}$ and $M_{2}^{\prime \prime}$ have rank $k$. Observe that $\mathcal{Z}(M)=\mathcal{Z}\left(M_{1}^{\prime \prime}\right) \cup \mathcal{Z}\left(M_{2}^{\prime \prime}\right)$. Using equation (2.1), it follows that a subset of $S_{1} \cup S_{2} \cup T_{1} \cup T_{2}$ is a basis of $M$ if and only if it is a basis of both $M_{1}^{\prime \prime}$ and $M_{2}^{\prime \prime}$. In particular, the constructions coincide when applied under the same set up, and the basis approach can be extended to cover the results in this paper. In the other direction, it is easy to check that if we replace inequality (3.1) with a slightly stronger inequality, then Theorem 3.1 applies even when $S_{1}$ and $S_{2}$ are not disjoint; of course, then we need $S_{1}^{\prime} \subseteq S_{1}-S_{2}$ and $S_{2}^{\prime} \subseteq S_{2}-S_{1}$. Likewise, Theorem 3.2 can be adapted (for instance, instead of $S_{1}-S_{1}^{\prime}$ on the right, we need $S_{1}-\left(S_{2} \cup S_{1}^{\prime}\right)$ ). Consistent with the hypotheses in Theorem 5.1 Theorem 3.3 also applies provided that $S_{1} \cap S_{2}$ is disjoint from $F I\left(M_{i}\right)$ and $F I\left(M_{i}^{*}\right)$ for $i \in\{1,2\}$, Thus, an advantage of dealing with disjoint ground sets is that it eliminates the need for assumptions about $F I\left(M_{i}\right)$ and $F I\left(M_{i}^{*}\right)$.

\section{ACKNOWLEDGEMENTS}

I am grateful to Jim Geelen for comments that led me to turn my attention to the topic of intertwines and for valuable feedback on this work. I thank Dirk Vertigan for providing copies of the slides of his talks on intertwines and spikes, and for permission to sketch his construction. I thank Anna de Mier for comments that improved the exposition.

\section{REFERENCES}

[1] J. Bonin and A. de Mier, The lattice of cyclic flats of a matroid, Ann. Comb., 12 (2008) 155-170.

[2] T. H. Brylawski, Constructions, in: Theory of Matroids, N. White, ed. (Cambridge University Press, Cambridge, 1986) 127-223.

[3] J. Geelen, Some open problems on excluding a uniform matroid, Adv. in Appl. Math. 41 (2008) 628-637.

[4] A. W. Ingleton, Transversal matroids and related structures, in: Higher combinatorics (Proc. NATO Advanced Study Inst., Berlin, 1976), Reidel, Dordrecht (1977) 117-131.

[5] J. G. Oxley, Matroid Theory, (Oxford University Press, Oxford, 1992). 
[6] H. Qin, Connected matroids with symmetric Tutte polynomials, Combin. Probab. Comput. 10 (2001) 179186.

[7] J. A. Sims, Some Problems in Matroid Theory, (Ph.D. Dissertation, Linacre College, Oxford University, Oxford, 1980).

Department of Mathematics, The George Washington University, Washington, D.C. 20052

E-mail address: jbonin@gwu.edu 